\title{
Liver Metastasis of Desmoplastic Small Round Tumour Treated with FOLFOX4 Combined with Anlotinib: A Case Report
}

\author{
Dong-Han Lin ${ }^{1}$, Feng Xia ${ }^{2}$, Lei Dou ${ }^{3}$, Yi-Fa Chen ${ }^{4, *}$ \\ 1,2,3,4 Department of Surgery, Tongji Hospital, Tongji Medical College, \\ Huazhong University of Science and Technology, Wuhan, China

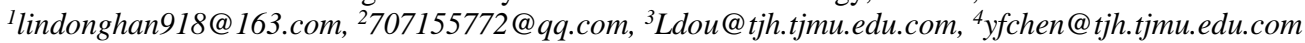 \\ *Correspondence Author
}

\begin{abstract}
Background: Desmoplastic small round cell tumor (DSRCT) is a rare, fast-growing neoformation occurred in younger populations, usually shown by involvement of abdomen and pelvis. Generally, DSRCT is similar to other cancers in manifestation. It can be distinguished from others by the applications of imageology, histology and molecular genetics. The patients discovered are usually at an advanced stage with metastases or corresponding complications. Here, we present a case of metastasis DSRCT of liver. Case report: The patient was a 41-year-old male with primary lesion in abdominopelvic region and multiple hepatic metastases which was diagnosed as DSRCT by imageology, immunohistochemical and histopathologic examinations and FISH experiment. The major treatment was FOLFOX4 combined with Anlotinib, and it achieved a fair result. Conclusion: The diagnosis of DSRCT is complicated and requires many methods to confirm it. For patients who are undergoing or going to implement the P6 regimen, with liver insufficiency or liver metastasis, FOLFOX4 combined with targeted drugs may be considered as an alternative to the P6 regimen.
\end{abstract}

Keywords: DSRCT, Liver metastasis, FOLFOX4, Anlotinib.

\section{Introduction}

Desmoplastic small round cell tumor (DSRCT) is a rare, highly malignant soft tissue neoplasm that was first reported by Greald[1] and Ordonez[2] in 1989 and officially named in 1991[3]. Predilection for younger populations, mostly in males aged 18 to 25 , with a male-female ratio $4: 1$ [4]. The etiology of the disease is uncertain. Ultrastructural and immunohistochemical studies have shown that the differentiation direction of tumor cells is unclear, but they are basically manifested as epithelial, muscle, neural differentiation in immunohistochemical reactivity, and it is currently believed to originate from mesothelial cells or stem cells with muti-directional differentiation potential[5-6]. DSRCT has a peculiar translocation, $\mathrm{t}(11 ; 22)(\mathrm{p} 13 ; 12)$, which forms an EWS-WT1 fusion gene[7]. Extra-peritoneal and extra-pelvic DSRCTs are infrequent, only a few cases described in testis[8], ethmoid sinus[9], lung[10], etc.

\section{Case Report}

A 42-year-old man complained of paroxysmal pain in the left lower abdomen and the low back for more than 20 days. The laboratory examination showed that CA125 was $386 \mathrm{U} / \mathrm{ml}$. Abdominal CT showed that there were space-occupying lesions in the left lower abdomen and pelvis, involving the left ureter and there were multiple progressive enhancement hepatic metastases. The patient underwent abdominal needle biopsy of the lesions and the extracts were subjected to immunohistochemical and histopathologic examinations and FISH experiment, which were diagnosed to be DSRCT. Genetic testing was also proceeded. After 1 cycle of P6 neoadjuvant chemotherapy, due to the intolerance of this regimen, the patient adopted to a regimen of FOLFOX4 combined with Anlotinib for 11 cycles. By this time, MRI of his abdomen showed that the hepatic metastases were similar to initial consultancy in size, and the retroperitoneal and pelvic nodules were significantly smaller than before.

\subsection{Imaging Findings}

At the first consultancy, the sagittal plane of magnetic resonance imagings of abdomen showed a high-intensity, unclear boundary mass in the left abdominopelvic region, which estimated to be $12.3 * 14.3 \mathrm{~cm}$, with multiple high-enhanced hepatic metastases (Figure 1a-b). T2-weighted magnetic resonance coronal plane imaging showed a high density annulus in the left lower quadrant $(7.7 * 6.5 \mathrm{~cm})$ and several hyper-enhanced lesions of which the biggest is $5.5 * 5.0 \mathrm{~cm}$ in the liver (Figure $1 \mathrm{c}-\mathrm{d}$ ).
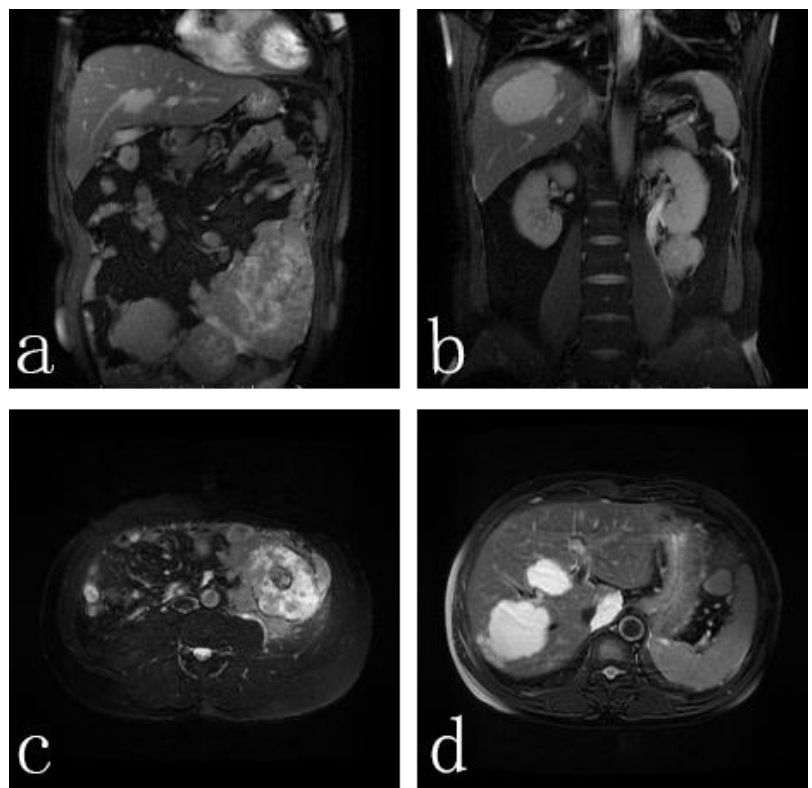

Figure 1: (a) The sagittal plane of MRI of abdomen. (b) The sagittal plane of MRI of hepatic metastases. (c) T2-weighted coronal plane of MRI of primary lesion. (d) T2-weighted coronal plane of MRI of hepatic metastases 
After 11 cycles of FOLFOX4 combined with Anlotinib, T2-weighted magnetic resonance coronal plane imaging of abdomen showed a high density $6.0 * 6.9 \mathrm{~cm}$ ring and hyper-enhanced lesions of which the biggest is $5.5 * 4.3 \mathrm{~cm}$ in the liver (Figure 2a-b). As we see, the retroperitoneal and pelvic nodules were significantly smaller than before and the hepatic metastases were similar to initial consultancy in size.

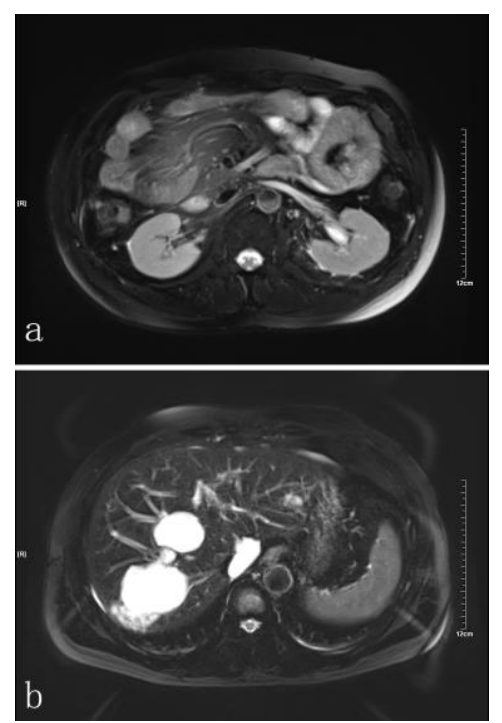

Figure 2: (a) T2-weighted coronal plane MRI of primary lesion. (b) T2-weighted coronal plane MRI of liver metastases

\subsection{Histological Findings}

At low magnification, there are small, round, and irregular neoplastic cells in forms of nests, cords, or sheets, surrounded by fibrillar connective tissue (Figure 3a). At high magnification, they are small, round convergent undifferentiated cells, with narrow cytoplasm and hyperchromatic nuclei (Figure 3b).

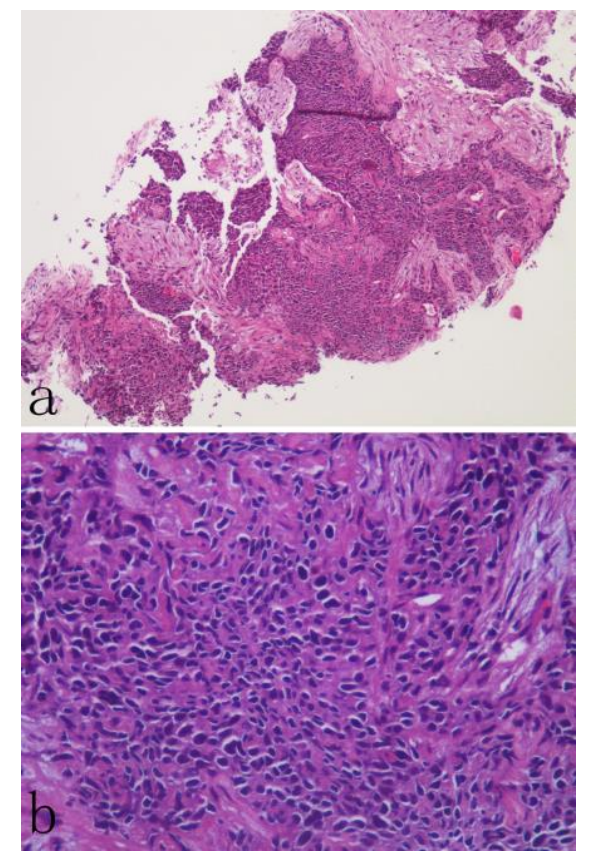

Figure 3: (a) $\mathrm{H} \& \mathrm{E} \times 10$, (b) $\mathrm{H} \& \mathrm{E} \times 40$

\subsection{Immunohistochemical and Genetics findings}

This immunohistochemical study was performed by using formalin-fixed paraffin embedded (FFPE) tumor sections. The tumor cells were positive for PCK, EMA, CK8/18, CK19,
Desmin, CD56, TLE1 (scattered positive) and negative for CD99, Syn, S-100, CgA, Hepatocyte, Glypican-3, Arginase-1, Caldesmon, Myogenin, CDX2, Villin (Figure 4a-f). The $\mathrm{Ki}-67$ hot spot region of $\mathrm{Li}$ was almost $50 \%$ (Figure $4 \mathrm{~g}$ ). The dual-colored fluorescence in situ hybridization analysis was performed on FFPE tissue by using EWSR1 break-apart probes and EWSR 1 division signal was detected in $50 \%$ of the tumor cells (Figure 4h). We then used the Next Generation Sequencing method which depended on tissue and venous blood of the patient on Illumina platform that covered 556 tumor hotspot genes. The mutation test results showed 3.6 mutations $/ \mathrm{Mb}$ of immunotherapy-related tumor mutation burden (TMB), which was lower than reference value; microsatellite analysis: MSS; negative for PD-L1.

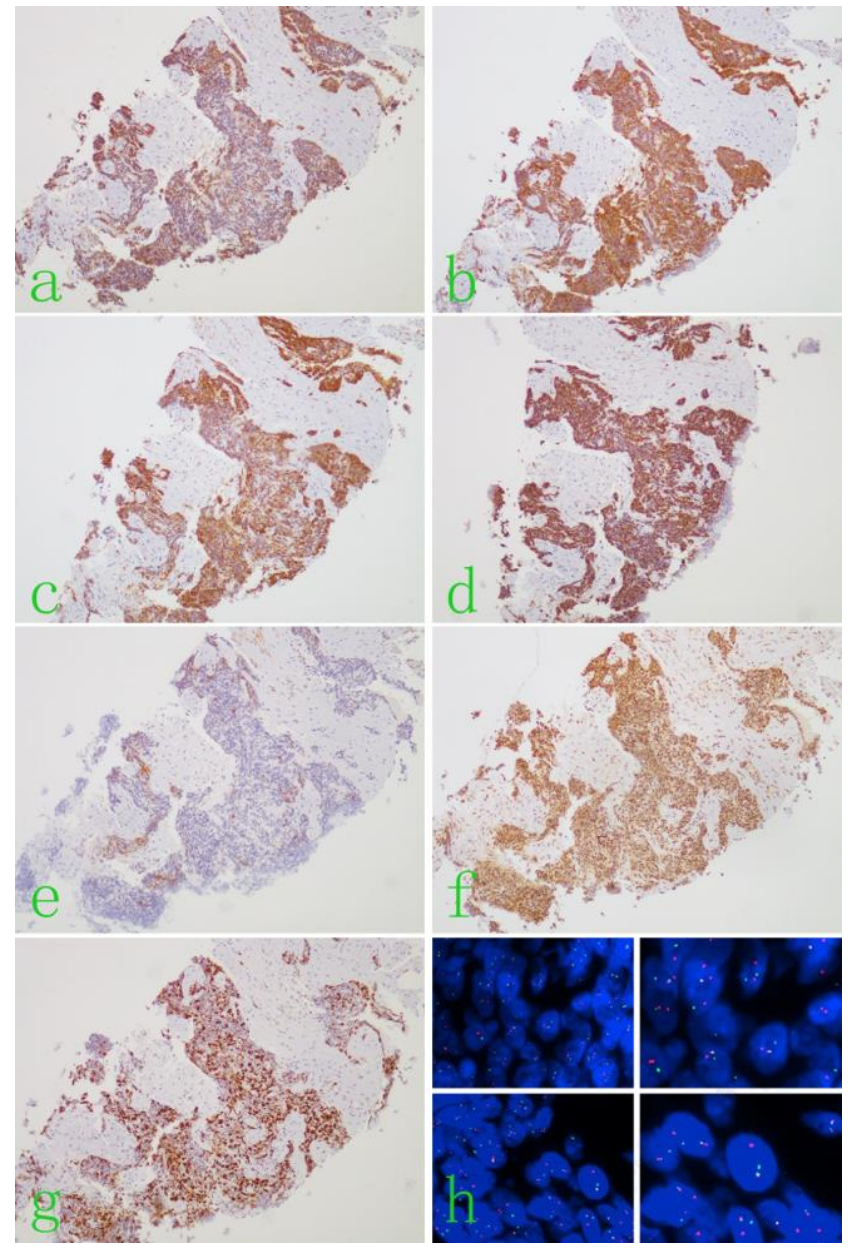

Figure4: (a) PCK, (b) CK8/18, (c) CK19, (d) Desmin, (e) CD56, (f) TLE-1,(g) Ki-67, (h) FISH experiment

\subsection{Follow-up}

Due to side effects of FOLFOX4, the patient was changed to a regimen of albumin Paclitaxel combined with Anlotinib for 4 cycles after accepting 11 cycles of FOLFOX 4 combined with Anlotinib, and his condition had no further progress.

Informed consent was obtained from the patient for the publication of the patient information and microscopic images.

\section{Discussion}

DSRCT is a rare, highly malignant neoplasm that was first reported by two boys in 1989[1,2]. DSRCTs are mainly 
shown by involvement of abdomen and pelvis, but Gerald et al. reported that $6 \%$ of DSRCTs can be found in extra-abdominal areas[11]. Radio-graphically, typical DSRCT as seen in this case usually shows multiple soft tissue masses with inhomogeneous density which may be accompanied by patchy hyperdensity shadows in the abdominopelvic region, involving the mesentery and omentum. Notably, the original focus is not located in any cavity or parenchymal organ and metastasis is often accompanied by corresponding symptoms which can be detected by imaging machine. Histologically, oval cells with desmoplastic and fibromyxoid stromata consist of nests surrounded by fibrillar connective tissue. Immunohistochemically, it is currently believed that DSRCT originated from mesothelial cells or primitive progenitor cells with multi-differentiation ability[6]. The overwhelming majority of the disease express mesenchymal, neurogenic and epithelial proteins simultaneously. Positive CK, NSE, EMA, Desmin, and Vimentin constituted an exceptional immunophenotype[5-6]. Genetically, the Ewing's sarcoma EWS gene on chromosome 22 is fused with the Wilms tumor WT1 gene on chromosome 11, $t(11,22)$ :(p13,q12), forming a unique EWS-WT1 protein[7]. Due to the lack of function of the WT1 tumor suppressor protein and the carcinogenic effect cased by the abnormal $59 \mathrm{kDa}$ fusion protein, resulting in nodules covering the intraperitoneal serosa or other areas[12]. In addition, FISH experiment of DSRCT reveals the separation of red and green signals.

DSRCT is a malignant tumor with a very low incidence and a high degree of malignancy. There is no standard unified treatment plan at present. Most patients died due to the rapid progress of the disease in a short time[13]. The treatment of DSRCT is similar to that of metastatic Ewing's sarcoma[12]. Cytotoxic chemotherapy and targeted drug therapy are usually used to inhibit or reverse growth of tumor, with the major purpose of reducing tumor-related symptoms and prolonging the survival of patients[12]. The treatment of the disease mainly include complete lesion resection, cytoreductive surgery, multimodal chemotherapy and adjuvant pelvic radiotherapy[14]. Only a few patients are treated with targeted drugs, myeloablative chemotherapy, and stem cell transplantation[15]. Because the disease usually involves extensively surrounding tissues, the resection is usually not complete[5]; The application of cytoreductive surgery can improve the survival of patients, but it cannot prolong the overall survival of patients[16]. For patients without distant metastases, complete resection or cytoreductive surgery should be the first option. For patients who are not suitable for surgery or have undergone metastasis and can tolerate chemotherapy drugs, the standard drugs combination of the P6 regimen chemotherapy, including Vincristine, Doxorubicin, Cyclophosphamide, Ifosfamide and Etoposide in combination or alternate use, or CEVAIE program (Ifosfamide, Vincristine, Actinomycin D, Carboplatin, Epirubicin, Etoposide), or VAIA program (Ifosfamide, Vincristine, Doxorubicin, Actinomycin, Bacteridin D) are usually adopted[17]. However, due to many adverse reactions related to treatment and the low effectiveness of this program, Doxorubicin and Ifosfamide are usually used as combination drugs[18]. Adjuvant radiotherapy is most commonly used to relieve the local symptoms of patients, and a few cases show that radiotherapy has a significant effect on the disease[19].
In this case, liver metastasis had existed when the disease was discovered. The diagnosis of DSRCT was based on imaging, immunohistochemistry, and genetic testing. Considering that cytoreductive surgery did not prolong the overall survival of the patient, and the patient was generally in good condition and could still tolerate chemotherapy, we applied the treatment with IE (Ifosfamide + Etoposide) in the P6 regimen. After 1 cycle of IE treatment, due to the poor physical tolerance of the patient, the regimen was changed to "FOLFOX4 combined with Anlotinib" and continued this treatment for 11 cycles. During the period, the primary lesion gradually shrank, but the metastasis did not change measurably. FOLFOX4 includes Oxaliplatin, Leucovorin and Fluorouracil. Oxaliplatin is the first platinum drug with significant on colon cancer, as a third-generation platinum-based chemotherapy drug, has relatively fewer adverse reactions and mild symptoms compared to the second-generation platinum-based chemotherapy drug Carboplatin. A study by Qin $\mathrm{S}$ et al. showed that the FOLFOX4 regimen containing Oxaliplatin is superior to the traditional chemotherapy drug Doxorubicin in terms of overall response rate, disease control rate, progression-free survival, and overall survival, and is tolerable and safe better[20]. For patients with metastatic colorectal cancer with liver insufficiency, FOLFOX is a safe option and no special dosage treatment is required[21]. It has good adaptability to the patient in this case. As a targeted drug, Anlotinib can effectively inhibit targets such as VEGF, PDGFR and c-Kit, and has anti-tumor angiogenesis and tumor growth inhibitory effects; it also has a good effect on soft tissue sarcoma[22]. There is no case report using "FOLFOX4 combined with targeted drug" to treat this disease. Due to individual differences, whether this regimen is effective for the patient population remains to be investigated. Although this program significantly relieved the patient's condition, due to prolonged use of multiple drugs, the patient experienced numbness in his hands and feet, and his physical condition became worse, so he changed to "albumin Paclitaxel combined with Anlotinib" to continue the treatment.

\section{Conclusion}

In summary, we have reported a liver metastasis of DSRCT treated with FOLFOX4 combined with Anlotinib. By imaging, immunohistochemistry, and genetic testing, the patient had the ultimate diagnosis. For patients who are not fit for cytoreductive surgery and can tolerate chemotherapy drugs, the P6 regimen chemotherapy would be a second option. For patients with liver insufficiency or liver metastasis, FOLFOX4 combined with targeted drugs may be considered as a replacement therapy to the $\mathrm{P} 6$ regimen.

\section{References}

[1] Gerald WL, Rosai J. Case 2. Desmoplastic small cell tumor with divergent differentiation. Pediatric pathology / affiliated with the International Paediatric Pathology Association. 1989; 9(2): 177-183.

[2] Ordonez NG, Zirkin R, Bloom RE. Malignant small cell epithelial tumor of the peritoneum coexpressing mesenchymal type intermediate filaments.AM J Surg Pathol. 1989; 13(5): 413. 
[3] Gerald, WL, Miller, HK, Battifora, H, Miettinen, M, Silva, EG, Rosai, J. Intra-abdominal desmoplastic small round-cell tumor. Report of 19 cases of a distinctive type of high-grade polyphenotypic malignancy affecting young individuals. Am J Surg Pathol. 1991; 15: 499-513.

[4] Thway K, Noujaim J, Zaidi S, et al. Desmoplastic small round cell tumor: pathology, genetics, and potential therapeutic strategies. Int J Surg Pathol. 2016; 24(8): 672-684.

[5] Kallianpur AA, Shukla NK, Deo SV, Yadav P, Mudaly D, Yadav R, Palaniappan RM. Updates on the multimodality management of desmoplastic small round cell tumor. J Surg Oncol. 2012; 105(6): 617-21.

[6] Ordóñez NG. Desmoplastic small round cell tumor: II: an ultrastructural and immunohistochemical study with emphasis on new immunohistochemical markers. Am J Surg Pathol, 1998; 22(11): 1314-1327.

[7] Hayes-Jordan A, LaQuaglia MP, Modak S. Management of desmoplastic small round cell tumor. Semin Pediatr Surg. 2016; 25(5): 299-304.

[8] He L, Wen S, Hu X, Guo C, Yi C. Primary desmoplastic small round cell tumor of the testis: A case report and review of the literature. Oncol Lett. 2013; 6(2): 565-567.

[9] Finke NM, Lae ME, Lloyd RV, Gehani SK, Nascimento AG. Sinonasal desmoplastic small round cell tumor: a case report. Am J Surg Pathol. 2002; 26(6): 799-803.

[10] Syed S, Haque AK, Hawkins HK, Sorensen PH, Cowan DF. Desmoplastic small round cell tumor of the lung. Archives of pathology \& laboratory medicine. 2002; 126(10): 1226-8.

[11] Gerald WL, Ladanyi M, de Alava E, Cuatrecasas M, Kushner BH, LaQuaglia MP, et al. Clinical, pathologic, and molecular spectrum of tumors associated with $\mathrm{t}(11 ; 22)(\mathrm{p} 13 ; \mathrm{q} 12)$ : desmoplastic small round-cell tumor and its variants. Journal of clinical oncology: official journal of the American Society of Clinical Oncology. 1998; 16(9): 3028-36.

[12] Subbiah V, Lamhamedi-Cherradi SE, Cuglievan B, Menegaz BA, Camacho P, Huh W, Ramamoorthy V, Anderson PM, Pollock RE, Lev DC, Qiao W, McAleer MF, Benjamin RS, Patel S, Herzog CE, Daw NC, Feig BW, Lazar AJ, Hayes-Jordan A, Ludwig JA. Multimodality Treatment of Desmoplastic Small Round Cell Tumor: Chemotherapy and Complete Cytoreductive Surgery Improve Patient Survival. Clin Cancer Res. 2018; 24(19): 4865-4873.

[13] Bono F, Testi MA, Rosai J. Desmoplastic small cell tumor with bizarre giant nuclei. Int J Surg Pathol. 2011; 19(6): 843-846.

[14] Honoré C, Amroun K, Vilcot L, et al. Abdominal desmoplastic small round cell tumor: multimodal treatment combining chemotherapy, surgery, and radiotherapy is the best option. Ann Surg Oncol. 2015; 22(4): 1073-1079.

[15] Cook RJ, Wang Z, Arora M, Lazarus HM, Kasow KA, Champagne MA, Saber W, van Besien KM, Hale GA, Copelan EA, Elmongy M, Ueno NT, Horn BN, Slavin S, Bishop MR, Stadtmauer EA. Clinical outcomes of patients with desmoplastic small round cell tumor of the peritoneum undergoing autologous HCT: a CIBMTR retrospective analysis. Bone Marrow Transplant. 2012; 47(11): 1455-8.
[16] Lal DR, Su WT, Wolden SL, Loh KC, Modak S, La Quaglia MP. Results of multimodal treatment for desmoplastic small round cell tumors. J Pediatr Surg. 2005; 40(1): 251-5.

[17] Kushner BH, LaQuaglia MP, Wollner N, Meyers PA, Lindsley KL, Ghavimi F, Merchant TE, Boulad F, Cheung NK, Bonilla MA, Crouch G, Kelleher JF Jr, Steinherz PG, Gerald WL. Desmoplastic small round-cell tumor: prolonged progression-free survival with aggressive multimodality therapy. J Clin Oncol 1996; 14(5): 1526-31.

[18] Bertuzzi A, Castagna L, Nozza A, et al. High-dose chemotherapy in poorprognosis adult small round cell tumours: clinical and molecular results from a prospective study[J]. J Clin Oncol, 2002; 20(8): 2181-2188.

[19] Pinnix CC, Fontanilla HP, Hayes-Jordan A, Subbiah V, Bilton SD, Chang EL, Grosshans DR, McAleer MF, Sulman EP, Woo SY, Anderson P, Green HL, Mahajan A. Whole abdominopelvic intensity-modulated radiation therapy for desmoplastic small round cell tumor after surgery. Int J Radiat Oncol Biol Phys. 2012; 83(1): 317-26.

[20] Qin S, Bai Y, Lim HY, et al. Randomized, multicenter, open label study of oxaliplatin plus fluorouracil / leucovorin versus doxorubicin as palliative chemotherapy in patients with advanced hepatocellular carcinoma from Asia. J Clin Oncol. 2013; 31(28): 3501-3508.

[21] Faugeras L, Dili A, Druez A, Krug B, Decoster C, D'Hondt L. Treatment options for metastatic colorectal cancer in patients with liver dysfunction due to malignancy. Crit Rev Oncol Hematol. 2017; 115: 59-66.

[22] Chi Y, Fang Z, Hong X, Yao Y, Sun P, Wang G, Du F, Sun Y, Wu Q, Qu G, Wang S, Song J, Yu J, Lu Y, Zhu X, Niu X, He Z, Wang J, Yu H, Cai J. Safety and Efficacy of Anlotinib, a Multikinase Angiogenesis Inhibitor, in Patients with Refractory Metastatic Soft-Tissue Sarcoma. Clin Cancer Res. 2018; 24(21): 5233-5238.

\section{Abbreviations}

- DSRCT:- Desmoplastic Small Round Cell Tumor

- EWS:- Ewing Sarcoma

- WT:- Wilms Tumor

- MRI:- Magnetic Resonance Imaging

- FFPE:- Formalin-fixed Paraffin Embedded

- FISH:- Fluorescence In Situ Hybridization

- CA125:- Cancer Antigen 125

- CT:- Computed Tomography

- MRI:- Magnetic Resonance Imaging

- PCK:- Pan Cytokeratin

- EMA:- Epithelial Membrane Antigen

- CK:- Cytokeratin

- SYN:- Synaptophysin

- CGA:- Chromogranin A

- TLE1:- Transducin-like Enhancer Of Split 1

- H\&E:- Hematoxylin-eosin 